\title{
ANALGESIA PREEMPTIVA EM ODONTOLOGIA
}

\section{PREEMPTIVE ANALGESIA IN DENTISTRY}

\author{
Katheryne Sue DeJean* Isis Rodrigues Menezes dos Santos* Fábio Vasconcelos \\ Andrade* Liane Maciel de Almeida Souza**
}

* Acadêmicos do curso de odontologia da Universidade Federal de Sergipe

** Prof. Msc. de Cirurgia e Anestesia da Universidade Federal de Sergipe

Recebido para publicação em 02/2008

Aceito para publicação em 08/2008

\section{RESUMO}

A lesão tecidual promove a sensibilização dos nociceptores periféricos (hiperalgesia) e o subsequente estímulo que amplifica a dor ao nível do sistema nervoso central. A analgesia preemptiva consiste em um tratamento farmacológico iniciado antes do procedimento cirúrgico, a fim de prevenir a hiperalgesia. Teoricamente, isso poderia ser mais eficaz se comparado a um regime analgésico introduzido após o término da intervenção, quando o processo inflamatório já se desencadeou. De fato, em medicina, já foram testados protocolos de analgesia preemptiva com alguns resultados favoráveis. Na área odontológica, os primeiros ensaios clínicos onde foi empregada essa "nova" modalidade de prevenção e controle da dor são mais ou menos recentes, ou seja, a maioria dos profissionais da área não tem conhecimento do assunto. Por esse motivo, nessa revisão da literatura, são apresentados e discutidos os resultados dos estudos realizados na clínica odontológica, quando a analgesia preemptiva foi empregada. Concluiu-se que ainda não há um consenso sobre a eficácia desse regime analgésico, bem como em relação aos fármacos de escolha, dosagens e posologias.

Palavras-Chave: Analgesia. Dor. Hiperalgesia

\begin{abstract}
The tissue injury promotes the sensitization of the peripheral nociceptives (hiperalgesia) and subsequent stimulation that amplifies the pain to the central nervous system. The preemptive analgesia consists of a pharmacological treatment initiated before the surgical procedure, in order to prevent the hiperalgesia. Theoretically, this could be more efficient if compared to an analgesic introduced after intervention, when the inflammatory process has already started. In fact, in Medicine, protocols of preemptive analgesia had been tested with some favorable results. In the Dentistry field, the first clinical attempts of this "new" means of prevention and pain control are recent, that is, the majority of the professionals are not aware of the subject. For this reason, in this literature review, the results of the studies carried through in the Dentistry practice are presented and discussed, when the preemptive analgesia was used. One may conclude that there isn't a consensus on the effectiveness of this analgesic regime, as well as the chosen drugs, dosages and posologies.
\end{abstract}

Keywords: Analgesia. Pain. Hiperalgesia. 


\section{Introdução}

Nos procedimentos odontológicos mais invasivos, a dor inflamatória se constitui no componente mais importante em resposta à injúria tecidual. (PINHEIRO et al., 2004). O termo "preemptiva" é uma tradução livre do termo inglês preemptive, e significa algo como eliminar o problema antes mesmo que ele apareça, ou nem dar a chance a um problema de sequer surgir. A ideia da analgesia preemptiva se baseia no fato de que a estimulação de fibras nociceptivas promove mudanças neurais e comportamentais, que podem persistir após a cessação do estímulo nocivo. Essa hipersensibilidade pós-traumática pode ser consequente a modificações no sistema nervoso periférico (hiperalgesia) ou central (hiperexcitabilidade). 1

O conceito de analgesia preemptiva é muito antigo e foi formulado por Crile (1913), a partir de observações clínicas. Ele defendeu a utilização de bloqueios regionais associados à anestesia geral, para impedir a dor causada por mudanças no sistema nervoso central durante a cirurgia, a partir dos estímulos nocivos ao cérebro. O ressurgimento desse conceito foi associado com uma série de estudos experimentais iniciados por Woolf em 1983 e destacados por Wall em 1988, na prevenção da dor pós-operatória. A partir disso, alguns ensaios de cunho clínico têm evidenciado a importância da analgesia preemptiva. (DAHL; KEHLET, 1993).

Acredita-se que essa estratégia, além de proporcionar conforto ao paciente, reduz o consumo de analgésicos para o controle da dor no período pós-operatório, o que abreviaria o tempo de recuperação do paciente. A adoção desse método como uma prática clínica padrão tem sido adiada, por controvérsias sobre se o regime farmacológico deve ser introduzido antes do trauma cirúrgico (analgesia preemptiva), após a lesão tecidual, mas antes do início da dor (analgesia preventiva) ou pela administração repetida de analgésicos de acordo com o curso pós-operatório. (PINHEIRO et al., 2004).

Este estudo tem como objetivo apresentar e discutir os estudos encontrados na literatura a respeito da aplicabilidade da analgesia preemptiva na Odontologia.

\section{Revisão da literatura}

A sensibilização central foi inicialmente estudada em vários modelos experimentais realizados em animais por Woolf em 1983. Wall (1988), foi o pioneiro em usar o termo "analgesia preemptiva”, quando observou a redução das alterações centrais, após a administração de analgésicos opioides e anestésicos locais, isoladamente ou associados, antes da incisão cirúrgica, havendo diminuição da intensidade da dor pós-operatória.

Estudos mostram que a anestesia geral promove a diminuição da transmissão dos impulsos dolorosos periféricos para o sistema nervoso central (SNC), porém não é capaz de inibi-los totalmente. (KATZ, 2001). Assim, estímulos nociceptivos provocam sensibilização central, que são responsáveis por aumento da intensidade da dor e do consumo de analgésicos no período pós-operatório. (OLIVEIRA et al., 2004). A intensidade da dor pós-operatória depende de fatores como: tipo e duração da cirurgia, extensão e natureza da lesão tecidual, atividade farmacológica do agente escolhido, analgesia adicional intra e pós-operatória. (AIDA et al., 1999; KATZ; McCARTNEY, 2002).

Na Odontologia, três grupos de fármacos são comumente empregados para controlar a dor: os anestésicos locais os anti-inflamatórios (AINES e corticosteroides) e os analgésicos de ação central e ou de ação periférica. (ROCHA et al., 2003).

A bupivacaína é um anestésico local de longa duração de ação, cuja administração proporciona analgesia eficaz durante as primeiras horas após uma intervenção cirúrgica (CAMPBELL et al., 1997). Por outro lado, um estudo recente parece ter demonstrado que a bupivacaína, em cirurgias de terceiros molares retidos, estimula a expressão do gene COX-2 (cicloxigenase-2) após a injúria tecidual, que é associada ao aumento da produção de prostaglandinas E2 (PGE2). Na prática, isso pode acarretar dor inflamatória aguda após a cessação dos efeitos desse anestésico. (GORDON et al., 2008).

Os analgésicos de ação periférica são inibidores da síntese de prostaglandinas, por meio da ação sobre as cicloxigenases (COX-1 e COX-2), o que resulta na atenuação da dor inflamatória 
aguda e também explica os efeitos adversos desse grupo de drogas. (KHAN et al., 2002, ROCHA et al., 2003).

Os analgésicos de ação central do tipo não opioides atuam por dois mecanismos: ligando-se com baixa afinidade a receptores u-opioides ou por inibição da realimentação de noradrenalina e serotonina. (WHITE, 2002, ROCHA et al., 2003). Os analgésicos opioides, por sua vez, são pouco empregados na clínica odontológica, pela baixa eficácia no controle de dores orofaciais. (MEHLIS$\mathrm{CH}, 2002)$.

Em um estudo randomizado duplo-cego, Bridgman et al. (1996), avaliou a eficácia analgésica do diclofenaco de sódio 100mg em exodontias de terceiros molares. Foi observado que a dor e o grau de abertura bucal após uma semana do procedimento cirúrgico não mostrou diferença significativa quanto a sua administração pré e pós-operatória, sugerindo assim, que essa droga anti-inflamatória não esteroidal não causa efeito analgésico preemptivo.

Zacharias et al. (1996), executaram extrações de terceiros molares em pacientes sob anestesia geral. Para o estudo, placebo, diclofenaco 100mg e metadona 10mg (opioide) foram administrados 60 a 90 minutos antes da cirurgia e em seguida, o grau de dor e a necessidade de medicação pós-operatória foram mensuradas durante três dias. Todos os pacientes receberam anestesia local e analgésicos durante o período operatório. Concluiu-se que a administração de drogas anti-inflamatórias não esteroidais e opioides não ofereceram efeito analgésico preemptivo nos pacientes que receberam analgesia adequada durante a cirurgia. O uso contínuo de drogas analgésicas no pós-operatório é talvez mais útil para esse propósito.

A administração de drogas que bloqueiam o estímulo nociceptivo diminuem a dor durante a fase de recuperação e, consequentemente, o menor consumo das mesmas. A adaptação desse método como prática clínica tem sido atrasada devido a controvérsias sobre se a intervenção farmacológica deve ser administrada antes do evento cirúrgico (analgesia preemptiva), antes do início da dor (analgesia preventiva), ou por sua repetida administração no pós-operatório. Dionne (2000) afirma que, todas essas medidas devem ser adotas na prática clínica com o intuito de minimizar o desenvolvimento da dor e do consumo de analgésicos, assim como, de acelerar a fase de recuperação.

De acordo com Kelly et al. (2001) as condições mais importantes para o estabelecimento de um efeito analgésico preemptivo eficiente consiste em administrar um nível ideal de antinociceptivo antes da injúria e manter este efeito analgésico na fase pós-operatória impedindo a sua sensibilização durante a fase inflamatória. A terapia do tipo multimodal mostra-se mais eficaz, pois produz poucos efeitos colaterais em comparação com altas doses do único agente.

López et al. (2005) compararam a eficácia analgésica do metilprednisolona (corticoide) e diclofenaco (AINES) após a remoção cirúrgica de terceiros molares. Pacientes foram separados em dois grupos aleatoriamente: um grupo do diclofenaco e um grupo do metilprednisolona. Os níveis da dor foram medidos em 1, 8, 24, 48 e 72 horas após. Houve menor dor no grupo do corticoide, mas isto não justifica seu uso rotineiro.

Micó-llorens et al. (2006) avaliaram a eficácia do metilprednisolona em dose única de $40 \mathrm{mg}$ no controle de complicações após exodontia de terceiros molares impactados. A cirurgia consistiu em osteotomia e odontosecção sob anestesia local. O grupo de estudo recebeu $40 \mathrm{mg}$ de metilprednisolona antes do procedimento e o grupo controle não. Os resultados confirmaram eficácia do metilprednisolona em fornecer um pós-operatório mais confortável quando administrado preemptivamente.

Ong e Tan (2004) compararam a eficácia analgésica do tramadol e ketorolac administrada na fase pré-operatória nas exodontias de terceiros molares. Os pacientes foram divididos em grupos equivalentes e de maneira aleatória. O Grupo I recebeu tramadol 50mg e o Grupo II ketorolac 30mg por via intravenosa antes da cirurgia. Após avaliação estatística observou-se que o ketorolac 30mg foi mais eficaz que o tramadol 50mg na prevenção da dor no pós-operatório.

ONG et al. (2004a) avaliaram a eficácia analgésica do ketorolac em cirurgias de terceiros molares mandibulares bilateralmente. Em cada 
paciente, um lado da mandíbula foi inicialmente tratado com ketorolac 30mg intravenoso antes do procedimento cirúrgico, seguido pela injeção de placebo após a cirurgia, enquanto que no lado oposto, procedeu-se na ordem inversa. Após 12 $\mathrm{h}$, os pacientes relataram que a intensidade da dor no lado que recebeu a administração de ketorolac 30mg no pré-operatório foi significativamente mais baixa quando comparado com o lado que recebeu essa droga no pós-operatório. O pré-tratamento com ketorolac intravenoso demonstrou ação analgésica pós-operátória eficiente para cirurgia de terceiros molares.

ONG et al. (2004b), investigaram o efeito analgésico do óxido nitroso em exodontias de terceiros molares inferiores simétricos e impactados no pré e pós operatório. Em um primeiro momento cirúrgico foi administrado óxido nitroso a $50 \%$ no pré-operatório durante 20 minutos e oxigênio a 100\% no pós-operatório por 20 minutos como placebo. No segundo momento, o lado oposto da mandíbula foi tratado na ordem inversa. Concluíram que o oxido nitroso a $50 \%$ não possui efeito analgésico para o controle da dor no pós-operatório quando administrado de maneira preemptiva.

Barron et al. (2004) avaliaram a ação da dexametasona e dipirona nas regiões inervadas pelo nervo alveolar inferior, infra-orbitário e lingual, após exodontias de terceiros molares. O grupo I recebeu dipirona e o grupo II dipirona e dexametasona. Todos os pacientes receberam a dose de dipirona no período pós-operatório. O tratamento pré-operatório com dexametasona associada a dipirona mostrou-se superior a dipirona isoladamente e impediu a hipersensibilidade sensorial após extração de terceiros molares.

A anestesia geral (AG) e a local (AL) evoluíram separadamente. Os procedimentos que não poderiam ser executados sob AL foram realizados sob AG. Nos anos 1980, estudos em animais sugeriram que o bloqueio periférico preemptivo de estímulos dolorosos ao sistema nervoso central com anestesia regional ou local e o uso de analgésicos não esteroidais poderia ser benéfico em atenuar a dor pós-operatória. Estudos clínicos baseados nesse princípio sugerem combinar a AL com AG, e talvez analgésicos não esteroidais com ou sem narcóticos para reduzir a severidade da dor pós-operatória. (KAUFMAN et al 2005).

Ong et al. 2005 avaliaram o efeito analgésico do rofecoxibe em exodontias. A primeira exodontia foi inicialmente tratada com rofecoxibe $50 \mathrm{mg}$ duas horas antes seguido pelo placebo 15 minutos após e em um segundo momento. O lado contralateral foi tratado com placebo duas horas antes seguido de rofecoxibe 50mg 15 minutos após cirurgia. Concluíram que o rofecoxibe apresenta boa eficácia analgésica para dor dentária aguda quando administrada na fase pré-operatória, reduzindo as exigências analgésicas dentro das primeiras 24 horas comparadas com a administração pós-operatória.

Young-Soo et al. 2005, compararam os efeitos analgésicos de um anti-inflamatório não esteroidal (AINE) para a dor cirúrgica oral em 3 intervalos diferentes de administração. O AINE foi testado 1 hora antes, 1 hora depois e sem administração. Observou-se que a dor foi mais prolongada no grupo que recebeu o AINE no pré-operatório em comparação aos demais. Conclui-se que o efeito analgésico do AINE administrado preemptivamente não é eficaz para o controle dor pós-operatória. Entretanto, os resultados implicam que os analgésicos administrados no pós-cirúrgico antes do desenvolvimento da dor são adequados para a analgesia sem a administração pré-operatória.

Young et al. (2006) compararam a eficácia do valdecoxibe em reduzir o incômodo causado pelo fio ortodôntico na fase preemptiva e pós. Três grupos foram selecionados: (I) placebo, (II) aqueles que receberam valdecoxibe $40 \mathrm{mg}$ de maneira preemptiva com pelo menos 30 minutos antes da colocação inicial do fio ortodôntico, ou (III) aqueles que receberam o valdecoxibe $40 \mathrm{mg}$ duas horas após a colocação do fio ortodôntico. No grupo II não houve aumento significativo no incômodo. $\mathrm{O}$ grupo I e III mostraram aumento significativo no incômodo após seis horas, com pico nas 24 horas. A analgesia preemptiva com drogas do tipo AINE podem evitar o incômodo associado ao tratamento ortodôntico.

Arantes (2006) avaliou, de forma comparativa, a eficácia do cetoprofeno e do parecoxibe. $\mathrm{Na}$ 
analgesia preemptiva, em 60 sujeitos submetidos à remoção de terceiros molares mandibulares inclusos. Os resultados mostraram que o parecoxibe, administrado antes do procedimento cirúrgico, foi mais eficaz que o cetoprofeno no controle da dor no tempo de 4h pós-operatórias, sendo menos efetivo decorrido 24h da intervenção.

\section{Discussão}

O controle da dor no pós-operatório é uma preocupação que aflige os cirurgiões-dentistas, e a busca de protocolos farmacêuticos que diminuam os transtornos causados pelas intervenções mais invasivas tem sido constante. No entanto, existe uma grande controvérsia de qual droga utilizar e como essa deve ser administrada. Nesse intuito, apresentam-se três correntes terapêuticas: a que defende o uso de fármacos no pré-operatório, a que somente utiliza a droga no pós-operatório e ainda a que utiliza a droga no pré, trans e pós-operatório.

A analgesia preemptiva consiste em um tratamento antinociceptivo que previne o estabelecimento da alteração do processamento central, o que amplifica a dor pós-operatória. (CRILE, 1913; WOOLF, 1983; KISSIN, 1986; DAHL; KEHLET, 1993; PINHEIRO, 2004; ANDRADE, 2006;).

Diante da literatura, a prevenção da dor pósoperatória é baseada em dois fenômenos, (1) o bloqueio efetivo do estímulo nocivo gerado durante um procedimento cirúrgico e durante o período inflamatório reduz subsequentemente a dor pósoperatória (fenômeno da analgesia preemptiva), e (2) um tratamento antinociceptivo iniciado antes do procedimento cirúrgico é muito mais efetivo na redução da dor pós-operatória do que aplicado depois do procedimento. (KISSIN, 1993).

Entretanto, Amantea et al. (1999) afirmam que, em alguns procedimentos cirúrgicos, a administração de somente uma droga analgésica antes da cirurgia, não é suficiente para produzir um efeito preemptivo. Assim sendo, o termo "analgesia preemptiva" como "analgesia dada antes da cirurgia” não seria adequado. Os autores sugerem que o conceito da prevenção da dor pós-operatória é mais bem definido pelo termo "analgesia preemptiva balanceada” que consiste em utilizar da analgesia em diferentes intervalos de tempo impedindo e controlando o estímulo doloroso durante todo procedimento: antes e/ou durante e, caso necessário, no período pós-operatório para a dor residual.

Estudos clínicos em Odontologia têm sido realizados buscando encontrar a droga ideal para um pós-operatório tranquilo e definir qual a corrente terapêutica mais adequada a seguir. Reuben e White (2002) e Jung et al. (2005) relatam que o uso oral e de maneira preemptiva dos AINES tem mostrado grande capacidade em fornecer analgesia pós-operatória eficaz. Andrade (2006) e Vane (1998) afirmam que dentre alguns fármacos utilizados na odontologia que previnem a sensibilização dos nociceptores por meio da inibição da cicloxigenase são os anti-inflamatórios não esteroidais cuja potência analgésica e anti-inflamatória varia de acordo com a meia-vida plasmática da droga e com a dose empregada. No entanto, se faz necessário definir qual o AINE a ser utilizado e em qual posologia.

Dentro desse grupo, as primeiras drogas a serem utilizadas nesse intuito foram o diclofenaco de sódio e os coxibes.

Analisando o efeito analgésico preemptivo do Diclofenaco de sódio 100mg, Zacharias et al. (1996) e Bridgman et al. (1996) relataram em seus estudos que a ação preemptiva do diclofenaco de sódio era inexistente e que a administração dessa droga imediatamente após a cirurgia controlava mais efetivamente a dor pós-cirúrgica.

López et al. (2005) e Micó-Llorens et al. (2006) afirmaram em seus estudos que o uso de corticoides no pré-operatório em dose única na odontologia tem mostrado bons resultados, promovendo um maior conforto no pós-operatório.

De acordo com Tonussi, em 1994, o diclofenaco seria uma droga que deprime diretamente o nociceptor, ou seja, ela atuaria prevenindo a sensibilização dos nociceptores e deprimindo sua atividade após estar sensibilizado, o que poderia explicar sua melhor eficácia no controle da dor já instalada.

Avaliando o efeito dos coxibes, Ong et al. (2005), Young et al. (2006), Arantes (2006) encon- 
traram efetividade analgésica nos coxibes testados quando utilizados de forma preemptiva.

Entretanto, existem estudos que sugerem um aumento de eventos cardiovasculares associados ao uso de coxibes. Por não existirem ensaios clínicos que comprovem a segurança dos coxibes, esse tipo de medicamento tem seu uso restrito. De uma maneira geral parece que os riscos de danos cardiovasculares estão relacionados à dose e ao tempo de uso dos coxibes. (ANDRADE, 2006). Sendo assim, o uso de coxibes de forma preemptiva, em cirurgias orais, não acarretaria danos ao sistema cardiovascular.

Outra droga bastante relatada na literatura como portadora de um efeito analgésico potente quando utilizada no pré-operatório é o anti-inflamatório esteroidal (corticoide). Barron et al. (2004) comprovou a efetividade analgésica da dexametasona quando utilizada de forma preemptiva em extrações de terceiros molares.

Andrade (2006) afirma que os corticosteroides, como a dexametasona e betametasona, são fármacos que previnem a sensibilização dos nociceptores por meio da inibição da fosfolipase A2. Além disso, são drogas de escolha na odontologia e que apresentam algumas vantagens em relação aos inibidores da cicloxigenase, entre elas: não apresentam efeitos colaterais significativos, não interferem na hemostasia, inibem a síntese de leucotrienos. Sua posologia é em dose única pré-operatória e o seu custo-benefício é mais vantajoso, uma vez que usados em única dose não causam efeitos de retardo cicatricial.

Apesar da comprovação da eficácia do uso preemptivo dos coxibes e dos corticosteroides na analgesia pós-operatória, ainda é cedo para se definir qual a droga e o esquema terapêutico que deva ser empregado como padrão nas cirurgias odontológicas.

\section{Conclusão}

A intensidade da dor pós-operatória, em geral, depende de fatores como o tipo e a duração da cirurgia, extensão e natureza da lesão tecidual, atividade farmacológica do(s) fármaco(s) empregado(s), e os momentos da sua administração (pré, intra e pósoperatória).

Ainda não há consenso na literatura no que diz respeito às drogas de eleição para o regime de analgesia preemptiva, bem como a dosagem posologia das mesmas.

\section{REFERÊNCIAS}

AIDA, S.; BABA, H.; YAMAKURA, T. The effectiveness of preemptive analgesia varies according to the type of surgery: a randomized, double-blind study. Anesth Analg, v.89, p.711-716, 1999.

AMANTEA, B.; GEMELLI, A.; MIGLIORINI, F.; TOCCI, R. Preemptive analgesia or balanced periemptive analgesia? Minerva Anestesiol. Jan-Feb., v.65, n.1-2, p.19-37, 1999

ANDRADE, E. D. Terapêutica medicamentosa em odontologia. 2. Ed.; Editora Artes Médicas, 2006.

ARAnTES, V. M. N. Analgesia preemptiva do cetoprofeno e do parecoxibe em cirurgia para remoção de terceiros molares inclusos. 2007.

BARRON, R. P.; BENOLIEL, R.; ZELTSER, R.; ELIAV, E.; NAHLIELI, O.; GRACELY, R. H. Effect of dexamethasone and dipyrone on lingual and inferior alveolar nerve hypersensitivity following third molar extractions: preliminary report. J. Orofac Pain, v.18, n.1, p.62-68, 2004.

BRIDGMAN, J. B.; GILLGRASS, T. G.; ZACHARIAS, $M$. The absence of any pre-emptive analgesic effect for nonsteroidal anti-inflammatory drugs. Br J. Oral Maxillofac Surg., v.34, n.5, Oct., p.428-431, 1996.

CAMPBELL W.I; RAMSAY-BAGGS P.; McMCAUGHEY $W$. The effect of pre-operative administration of bupivacaine compared with its postoperative use. Anaesthesia, v.52, n.12, p.1212-1216, Dec.,1997.

CRILE, G. W. The kinetic theory of shock and its prevention through anoci-association (shockless operation). Lancet, v.185, p.7-16, 1913.

DAHL, J. B.; KEHLET, H. The value of pre-emptive analgesia in the treatment of postoperative pain. Br $\mathbf{J}$ Anaesth., v.70, p.434-9, 1993.

GORDON S. M.; CHUANG, B. P.; WANG, X. M.; HAMZA, M. A.; ROWAN, J. S.; BRAHIM, J. S.; DIONNE, R. A. The differential effects of bupivacaine and lidocaine on prostaglandin E2 release, cyclooxygenase 
gene expression and pain in a clinical pain model. Anesth Analg.; v.106, n.1, p.321-7, Jan., 2008.

DIONNE, R. Preemptive vs preventive analgesia: which approach improves clinical outcomes? Compend Contin Educ Dent., v.21, n.1, p.48, 51-4, 56, Jan. 2000.

JUNG, Y. S.; KIM, M. K.; UM, Y. J. et al. The effects on postoperative oral surgery pain by varying NSAID administration times: comparison on effect of preemptive analgesia. doi:10.1016/j.tripleo.2005.02.065

KATZ, J. Pre-emptive analgesia: importance of timing. Can J Anaesth,; v.48, p.105-114, 2001.

.; MCCARTNEY, C. J. L. Current status of preemptive analgesia. Curr Opin Anaesthesiol, v.15, p.435441, 2002.

KAUFMAN, E.; EPSTEIN, J. B.; GORSKY, M.; JACKSON, D. L.; KADARI, A. Preemptive analgesia and local anesthesia as a supplement to general anesthesia. Anesth Prog. Spring, v.52, n.1, p.29-38, 2005.

KELLY, D. J.; AHMAD, M.; BRULL, S. J. Preemptive analgesia II: recent advances and current trends. Can J Anaesth. v.48, n.11, p.1091-101, Dec. 2001.

KHAN, A. A.; DIONNE, R. A. The COX-2 inhibitors: new analgesic and anti-inflammatory drugs. Dent Clin North Am, Philadelphia, v. 46, n. 4, p. 679-690, Oct. 2002.

KISSIN, I. Preemptive analgesia: why its effect is not always obvious. Anesthesiology, v.84, n.5, p.1015-1019, May 1996.

LÓPEZ-CARRICHES, C.; MARTÍNEZ-GONZÁLEZ, J. M.; DONADO-RODRÍGUEZ, M. Analgesic efficacy of diclofenac versus methylprednisolone in the control of postoperative pain after surgical removal of lower third molars. Med Oral Patol Oral Cir Bucal. v.10, n.5, p.432-9, Nov-Dec., 2005.

MEHLISCH, D. R. The efficacy of combination analgesic therapy in relieving dental pain. J. Am Dent Assoc, Chicago, v. 133, n. 7, p. 861-871, July 2002.

MICÓ-LLORENS, J. M.; SATORRES-NIETO, M.; GARGALLO-ALBIOL, J.; ARNABAT-DOMÍNGUEZ, J.; BERINI-AYTÉS, L.; GAY-ESCODA, C. Efficacy of methylprednisolone in controlling complications after impacted lower third molar surgical extraction. Eur J Clin Pharmacol.;v.62, n.9, p.693-8, Aug/Sep, 2006.

OLIVEIRA, C. M. B.; SAKATA, R. K.; ISSY, A. M.; GARCIA, J. B. S. Cetamina e analgesia preemptiva. Rev Bras Anestesiol, v.54, n.5, p.739 - 752, 2004.

ONG, K. S.; TAN, J. M. Preoperative intravenous tramadol versus ketorolac for preventing postoperative pain after third molar surgery. Int J Oral Maxillofac Surg.; v.33, n.3, p.274-8. Apr, 2004.

ONG, K. S.; SEYMOUR, R. A.; CHEN, F. G.; HO, V. C. Preoperative ketorolac has a preemptive effect for postoperative third molar surgical pain. Int $\mathbf{J}$ Oral Maxillofac Surg. v.33, n.8, p.771-6, Dec., 2004a.

.; TAN, J. M. A prospective randomized crossover study of the preemptive analgesic effect of nitrous oxide in oral surgery. Oral Surg Oral Med Oral Pathol Oral Radiol Endod.; v.98, n.6, p.637-42, Dec., 2004b.

.; YEO, J. F.; HO, K. H.; LIRK, P. The efficacy of preoperative versus postoperative rofecoxib for preventing acute postoperative dental pain: a prospective randomized crossover study using bilateral symmetrical oral surgery. Clin J Pain. v.21, n.6, p.536-42, Nov./Dec., 2005.

PINHEIRO, M. L. P., TAMINATO, R. L., MOREIRA A., ANDRADE, E. D. Analgesia preemptiva em odontologia. Braz J Oral Sci. v.3, n.10; Abstracts - II International Dental Meeting - UNICAMP and XI Dental Meeting of Piracicaba, 2004.

ROCHA, R. G.; TORTAMANO, N.; ADDE, C. A.; SIMONE, J. L.; PEREZ, F. E. G. O controle da dor em odontologia através da terapêutica medicamentosa. In: CONCLAVE ODONTOLÓGICO INTERNACIONAL DE CAMPINAS, 15. Anais... n.104 - Mar/Abr., 2003.

REUBEN, S. S.; BHOPATKAR, S.; MACIOLEK, H. The preemptive analgesic effect of rofecoxib after ambulatory arthroscopic knee surgery. Anesth Analg., v.94, p.55-59, 2002.

TONUSSI C. R., FERREIRA S. H. MEchanism of diclofenac analgesia: direct blokade of inflammatory sensitization. Eur J Pharmacol, v.251, p.173-9,1994.

VANE, J. R. BOTTING, R. M. Mechanism of action of nonsteroidal anti-inflammatory drugs. Am J Med 1998104 (3A): 2S-8S; Discussion 21S-22S.

WALL, P. D. The prevention of postoperative pain. Pain, v.33, p.289-290, 1988.

WHITE, P. F. The role of non-opioid analgesic techniques in the management of pain after ambulatory surgery. Anesth Analg., v.94, p.577-585, 2002.

WOOLF, C. J. Evidence for a central component of post-injury pain hypersensitivity. Nature,v.306, p.686688,1983.

YOUNG-SOO, JUNG; DDS, MOON-KEY KIM DDS, YOO JUNG UM, HYUNG-SIK PARK DDS, MSD, EUIWUNG LEE DDS, AND JEONG-WAN KANG MD. 
The effects on postoperative oral surgery pain by varying NSAID administration times: Comparison on effect of preemptive analgesia. Oral Surg Oral Med Oral Pathol Oral Radiol Endod., v.100, n.5, p.559-63, Nov., 2005.

YOUNG, A. N.; TAYLOR, R. W.; TAYLOR, S. E.; LINNEBUR, S. A.; BUSCHANG, P.H. Evaluation of preemptive valdecoxib therapy on initial archwire placement discomfort in adults. Angle Orthod., v.76, n.2, p.251-9, Mar. 2006.

ZACHARIAS, M.; HUNTER, K. M.; BAKER, A. B. Effectiveness of preoperative analgesics on postoperative dental pain: a study. Anesth Prog., v.43, n.3, p.92-6, Summer 1996. Disponível em: <www. dor. med. br/ dorclinica/dor.htm>. Acesso em: 28 ago.2008. 